\title{
Effects of Crack Closure and Friction for Linear Crack under Normal Impact
}

Oleksandr V. Menshykov", Marina V. Menshykova, Igor A. Guz

Centre for Micro- and Nanomechanics (CEMINACS)

School of Engineering, University of Aberdeen

Aberdeen AB24 3UE, Scotland, UK

\begin{abstract}
This paper concerns the fracture mechanics problem for elastic cracked materials under transient dynamic loading. The nonlinear contact problem for a linear crack under normal impact loading is solved by the boundary integral equations in the frequency domain and the components of the solution are presented by the Fourier exponential series. The algorithm convergence is proved and the solution is obtained for different friction coefficients. The dynamic stress intensity factors are computed for different stress pulses and compared with those obtained neglecting the crack closure and friction.
\end{abstract}

Keywords: Nonlinear Fracture Mechanics, Cracked Materials, Impact Load, Linear Crack, Contact Interaction, Friction.

*Corresponding author. Tel.: +44 1224 273326; fax: +43 1224 272519;

e-mail: $\underline{0 . m e n s h y k o v @ a b d n . a c . u k}$ 


\section{Introduction}

Crack-like defects in engineering materials being subjected to any type of loading could be the main reason of the structure collapse. When the material is subjected to the dynamic, particularly impact loading, the elastic waves are generated within the structure, and refraction and reflection of these waves cause the local stresses to increase above the static values, making the problem of high importance.

To tackle the problem of normal impact loading of the faces of the crack Freund [1] proposed to use the fundamental solution for a plane strain problem of a half-plane crack situated in unbounded elastic solid, and focused on the distribution of the stress intensity factors in time. Ma and Hou [2, 3] extended the Freund methodology to study the transient effect on the dynamic in-plane and anti-plane crack problems presenting the time-dependent full-field solutions of stresses and stress intensity factors. The characteristic time was found, so the static solutions can be used as approximations to the associate dynamic problems.

Collinear cracks in homogeneous linearly elastic material subjected to impact loading were considered in [4]. To solve the problem for equally spaced cracks the integral equations based on the dislocation fundamental solution were derived. The equations were solved in the Laplace transform domain using the Gauss integration quadrature and then inverted to calculate the stress intensity factors in the time domain. Multiple cracks in homogeneous linearly elastic plane under anti-plane dynamic loading solved in [5]. To calculate the dynamic stress intensity factors for each crack tip the inverse Laplace scheme was implemented. Numerical examples were given for several parallel cracks under normally incident of a shear stress wave. The problem for multiple curved cracks under anti-plane shear impact loading was studied in [6], where for an infinite isotropic plane weakened by multiple smooth cracks 
the distributed dislocation method was used to construct singular integral equations. The dislocation density on the faces of the cracks could be numerically computed and then used to determine stress intensity factors. The investigation on the influence of such parameters as the time, crack pattern and crack length on the dynamic stress intensity factors was carried out.

Crack/surface intersection problem for the impact loading was solved in [7] using time-domain boundary element method. Influence of free-surface on dynamic stress intensity factors for a central crack under the step loading was investigated. In the paper the critical intersection angle of the crack front with the free-surface was estimated.

2-D transient dynamic crack problems for homogeneous and piezoelectric solids using boundary integral equation method were solved in [8, 9]. The dynamic stress intensity factors were calculated for the linear finite length crack in infinite body under impact loading, where regular integrals were calculated numerically and singular and hypersingular integrals were integrated analytically. Comparison of two hypersingular time domain boundary element methods was done in [10]. In the paper the problem of linear crack under transient load situated in two-dimensional, homogeneous, anisotropic and linear elastic solid was considered. The extensive numerical tests were carried out to compare the accuracy, efficiency and stability of the two different time domain boundary element methods.

The comparison of time domain and frequency domain boundary element methods was carried out in [11]. Udzava's type iterative algorithm and boundary element methods were used for two-dimensional contact problem solution in which the linear crack faces' contact interaction is taken into account under the harmonic loading. In the paper the results for crack opening, contact forces and dynamic stress 
intensity factors were obtained. It was also noted that the crack face contact interaction must be taken into account, as it changes the distribution of the stresses and the displacements not only quantitatively but also qualitatively [12]. The contact forces and the displacement discontinuity on the crack faces are studied in [13] for the harmonic loading of the penny shaped crack. Considering the oblique harmonic loading of the linear crack, the effect of the friction between the crack faces was extensively studied [14]. The analysis of the results obtained for different angles of wave incidence, the wave frequencies and friction coefficients was carried out.

Contact problem for the interface linear crack was solved in [15] for normal tension-compression load. In the paper the multi-parametric iterative algorithm was presented and its convergence was investigated. Also, the system of boundary integral equations for displacements and tractions was derived and the effect of frequency on stress intensity factors for opening and transverse shear modes was considered. Interfacial penny-shaped crack under harmonic loading was considered in [16]. Furthermore, the boundary integral equation method in the frequency domain was used for the first time to solve of problem for a linear crack under transient load [17]. In the paper the dynamic stress intensity factors are computed for different stress pulses, including the Heaviside step loading.

In the current study the approach presented in [17] is combined with the iterative algorithm [14] for the case of a linear crack under normal transient loading. The nonlinear effects of the crack closure and friction are analysed for the first time and the convergence of the algorithm is investigated. 


\section{Methodology}

Let us consider the linearly elastic cracked material under external loading. The behaviour of the body satisfies the equations of motion (the body forces are absent) $[1,18]:$

$$
\partial_{j} \sigma_{i j}(\mathbf{x}, t)=\rho \partial_{t}^{2} u_{i}(\mathbf{x}, t), \mathbf{x} \in V, t \in \mathfrak{I}=[0, T]
$$

and the generalized Hooke's law

$$
\sigma_{i j}(\mathbf{x}, t)=C_{i j k l} \varepsilon_{k l}(\mathbf{x}, t), \mathbf{x} \in V, t \in \mathfrak{J}
$$

subject to some boundary conditions. In Eqs. (1) and (2) the components of the displacements and stresses are denoted as $u_{i}(\mathbf{x}, t)$ and $\sigma_{i j}(\mathbf{x}, t), \rho$ is the mass density, $C_{i j k l}$ is the elasticity tensor and the components of the Cauchy strain tensor are given by $\varepsilon_{k l}(\mathbf{x}, t)=\left(\partial_{k} u_{l}(\mathbf{x}, t)+\partial_{l} u_{k}(\mathbf{x}, t)\right) / 2$.

For an isotropic material Eqs. (1) and (2) lead to the following linear Lamé equations of elastodynamics for the displacement field:

$$
\mu \delta_{i j} \frac{\partial}{\partial x_{k}} \frac{\partial}{\partial x_{k}} u_{j}(\mathbf{x}, t)+(\lambda+\mu) \frac{\partial}{\partial x_{i}} \frac{\partial}{\partial x_{j}} u_{j}(\mathbf{x}, t)=\rho \frac{\partial}{\partial t} \frac{\partial}{\partial t} u_{i}(\mathbf{x}, t), \mathbf{x} \in V, t \in \mathfrak{I},
$$

here $\delta_{i j}$ is the Kronecker delta, $\mu$ and $\lambda$ are the Lamé elastic constants.

With the assumption of zero initial displacements of the points of the body, what means that the body is free of strain at the initial moment, the initial conditions are as follows:

$$
\mathbf{u}^{(m)}(\mathbf{x}, 0)=\operatorname{grad} \mathbf{u}^{(m)}(\mathbf{x}, 0)=0, \quad \mathbf{x} \in V .
$$

The conditions of continuity for displacements and stresses are satisfied at bonding interfaces:

$$
\mathbf{u}^{(+)}(\mathbf{x}, t)=\mathbf{u}^{(-)}(\mathbf{x}, t), \quad \boldsymbol{\sigma}^{(+)}(\mathbf{x}, t)=\boldsymbol{\sigma}^{(-)}(\mathbf{x}, t), \quad \mathbf{x} \in \Gamma^{\mathrm{int}}, \quad t \in \mathfrak{I},
$$


In the case of elastic solid occupying the whole space, the Sommerfeld radiation-type condition, which provides a finite elastic energy of an infinite body, is also imposed at infinity on the vector of displacements:

$$
\|\mathbf{u}(\mathbf{x}, t)\| \leq c / r,
$$

where $c$ is a constant and $r \rightarrow \infty$ is the distance from the origin.

The components of displacement field in terms of boundary displacements and tractions using the Somigliana dynamic identity could be represented in 2D case as follows:

$$
\begin{gathered}
u_{j}^{(m)}(\mathbf{x}, t)=\int_{\mathrm{T}} \int_{\Gamma^{(m)}}\left(t_{i}^{(m)}(\mathbf{y}, \tau) U_{i j}^{(m)}(\mathbf{x}, \mathbf{y}, t-\tau)-u_{i}^{(m)}(\mathbf{y}, \tau) W_{i j}^{(m)}(\mathbf{x}, \mathbf{y}, t-\tau)\right) d \mathbf{y} d \tau, \\
\mathbf{x} \in \Omega^{(m)}, \quad t \in \mathfrak{I}, \quad j=\overline{1,2}
\end{gathered}
$$

where the fundamental displacement is

$$
U_{i j}^{(m)}(\mathbf{x}, \mathbf{y}, t-\tau)=\frac{1}{2 \pi \mu^{(m)}}\left(\psi^{(m)} \delta_{i j}-\chi^{(m)} \frac{\left(y_{i}-x_{i}\right)}{r} \frac{\left(y_{j}-x_{j}\right)}{r}\right),
$$

$r$ is the distance between the observation point and the load point, $W_{i j}^{(m)}(\mathbf{x}, \mathbf{y}, t-\tau)=C_{i j k l} e_{j}(\mathbf{y}) \partial_{l} U_{k j}^{(m)}(\mathbf{x}, \mathbf{y}, t-\tau)$ is the traction fundamental solution; $\Gamma^{(m)}$ is the subdomain boundary, which can conclude parts of the external boundary, bonding interfaces, $\Gamma^{\text {int }}$, and cracks' surfaces, $\Gamma^{c r}$; and functions $\psi^{(m)}$ and $\chi^{(m)}$ for the case considered will be defined later.

Applying the differential operator

$$
P_{i k}[\bullet,(\mathbf{y})]=\lambda n_{i}(\mathbf{y}) \frac{\partial[\bullet]}{\partial y_{k}}+\mu\left[\delta_{i k} \frac{\partial[\bullet]}{\partial \mathbf{n}(\mathbf{y})}+n_{k}(\mathbf{y}) \frac{\partial[\bullet]}{\partial y_{i}}\right]
$$

one can obtain from $U_{i j}^{(m)}(\mathbf{x}, \mathbf{y}, t-\tau)$ the following integral kernel 


$$
\begin{aligned}
& W_{i j}^{(m)}(\mathbf{x}, \mathbf{y}, t-\tau)=\lambda^{(m)} n_{i}^{(m)}(\mathbf{y}) \frac{\partial}{\partial y_{k}} U_{k j}^{(m)}(\mathbf{x}, \mathbf{y}, t-\tau)+ \\
& \mu^{(m)} n_{k}^{(m)}(\mathbf{y})\left[\frac{\partial}{\partial y_{k}} U_{i j}^{(m)}(\mathbf{x}, \mathbf{y}, t-\tau)+\frac{\partial}{\partial y_{i}} U_{k j}^{(m)}(\mathbf{x}, \mathbf{y}, t-\tau)\right] .
\end{aligned}
$$

Then the integral representation of the components of the traction field can be also derived from the Somigliana identity (7):

$$
\begin{gathered}
p_{j}^{(m)}(\mathbf{x}, t)=\int_{T} \int_{\Gamma^{(m)}}\left(p_{i}^{(m)}(\mathbf{y}, \tau) K_{i j}^{(m)}(\mathbf{x}, \mathbf{y}, t-\tau)-u_{i}^{(m)}(\mathbf{y}, \tau) F_{i j}^{(m)}(\mathbf{x}, \mathbf{y}, t-\tau)\right) d \mathbf{y} d \tau, \\
\mathbf{x} \in \Omega^{(m)}, \quad t \in T, \quad j=1,2
\end{gathered}
$$

where integral kernels $K_{i j}^{(m)}(\mathbf{x}, \mathbf{y}, t-\tau)$ and $F_{i j}^{(m)}(\mathbf{x}, \mathbf{y}, t-\tau)$ can be obtained from kernels $U_{i j}^{(m)}(\mathbf{x}, \mathbf{y}, t-\tau)$ and $W_{i j}^{(m)}(\mathbf{x}, \mathbf{y}, t-\tau)$ using the differential operator (9) with respect to $\mathbf{x}$.

We assume that that the distributions of boundary displacements and traction vectors are smooth enough. Then for the limiting case $\mathbf{x} \rightarrow \Gamma^{(m)}$ it is possible to deduce the boundary integral equations connecting the tractions and the displacements on the boundaries of all subdomains. Using the conditions of continuity for displacements and stresses at bonding interfaces the resulting system of boundary integral equations for the whole body can be obtained:

$$
\begin{aligned}
& \frac{1}{2} u_{j}^{(m)}(\mathbf{x}, t)=\int_{T} \int_{\Gamma^{(m)}}\left(p_{i}^{(m)}(\mathbf{y}, \tau) U_{i j}^{(m)}(\mathbf{x}, \mathbf{y}, t-\tau)-u_{i}^{(m)}(\mathbf{y}, \tau) W_{i j}^{(m)}(\mathbf{x}, \mathbf{y}, t-\tau)\right) d \mathbf{y} d \tau, \\
& \frac{1}{2} p_{j}^{(m)}(\mathbf{x}, t)=\int_{T} \int_{\Gamma^{(m)}}\left(p_{i}^{(m)}(\mathbf{y}, \tau) K_{i j}^{(m)}(\mathbf{x}, \mathbf{y}, t-\tau)-u_{i}^{(m)}(\mathbf{y}, \tau) F_{i j}^{(m)}(\mathbf{x}, \mathbf{y}, t-\tau)\right) d \mathbf{y} d \tau,
\end{aligned}
$$

where $\mathbf{x} \in \Gamma^{(m)}, \quad t \in T$.

As it was shown in [17], if the transient loading and the normal and tangential components of the displacement discontinuity vector and the traction vector are approximated by the trigonometric Fourier series with respect to the time 


$$
\begin{gathered}
p_{j}(\mathbf{x}, t)=\frac{p_{j, \cos }^{0}(\mathbf{x})}{2}+\sum_{k=1}^{+\infty}\left(p_{j, \cos }^{k}(\mathbf{x}) \cos \left(\omega_{k} t\right)+p_{j, \sin }^{k}(\mathbf{x}) \sin \left(\omega_{k} t\right)\right), \\
{\left[u_{j}(\mathbf{x}, t)\right]=\frac{\left[u_{j, \mathrm{cos}}^{0}(\mathbf{x})\right]}{2}+\sum_{k=1}^{+\infty}\left(\left[u_{j, \cos }^{k}(\mathbf{x})\right] \cos \left(\omega_{k} t\right)+\left[u_{j, \sin }^{k}(\mathbf{x})\right] \sin \left(\omega_{k} t\right)\right),}
\end{gathered}
$$

where $\mathbf{x} \in \Omega, \omega_{k}=2 \pi k / T, j=1,2$ and

$$
\begin{aligned}
& p_{j, \mathrm{cos}}^{k}(\mathbf{x})=\frac{\omega}{2 \pi} \int_{0}^{T} p_{j}(\mathbf{x}, t) \cos \left(\omega_{k} t\right) d t, \quad p_{j, \sin }^{k}(\mathbf{x})=\frac{\omega}{2 \pi} \int_{0}^{T} p_{j}(\mathbf{x}, t) \sin \left(\omega_{k} t\right) d t, \\
& {\left[u_{j, \mathrm{cos}}^{k}(\mathbf{x})\right]=\frac{\omega}{2 \pi} \int_{0}^{T}\left[u_{j}(\mathbf{x}, t)\right] \cos \left(\omega_{k} t\right) d t, \quad\left[u_{j, \sin }^{k}(\mathbf{x})\right]=\frac{\omega}{2 \pi} \int_{0}^{T}\left[u_{j}(\mathbf{x}, t)\right] \sin \left(\omega_{k} t\right) d t,} \\
& k \in N_{0}=0,1, \ldots,+\infty,
\end{aligned}
$$

then the method and techniques developed in [11-18] may be used to solve the problem in the time domain.

In the considered homogeneous case the system of boundary integral equations (12)-(13) can be further reduced to the following:

$$
\begin{aligned}
& p_{j, \cos }^{k}(\mathbf{x})-i p_{j, \sin }^{k}(\mathbf{x})= \\
& -\sum_{m=1}^{2} \int_{\Omega}\left(F_{m j}^{\mathrm{Re}}\left(\mathbf{x}, \mathbf{y}, \omega_{k}\right)+i F_{m j}^{\mathrm{Im}}\left(\mathbf{x}, \mathbf{y}, \omega_{k}\right)\right)\left(\Delta u_{m, \cos }^{k}(\mathbf{y})-i \Delta u_{m, \sin }^{k}(\mathbf{y})\right) d \mathbf{y}, \\
& \mathbf{x} \in \Omega, \quad k \in N_{0}, \quad j=1,2,
\end{aligned}
$$

where $i$ is the imaginary unit; $F_{m j}^{\mathrm{Re}}\left(\mathbf{x}, \mathbf{y}, \omega_{k}\right)$ and $F_{m j}^{\mathrm{Im}}\left(\mathbf{x}, \mathbf{y}, \omega_{k}\right)$ are the real and the imaginary parts of the integral kernel $F_{m j}\left(\mathbf{x}, \mathbf{y}, \omega_{k}\right)$. For a linear crack we can write them down as:

$$
\begin{gathered}
F_{12}\left(\mathbf{x}, \mathbf{y}, \omega_{k}\right)=F_{21}\left(\mathbf{x}, \mathbf{y}, \omega_{k}\right)=0 \\
F_{11}\left(\mathbf{x}, \mathbf{y}, \omega_{k}\right)=\frac{i \mu}{4}\left[\left(\frac{\omega_{k}}{c_{2}}\right)^{2} H_{0}^{(1)}\left(\frac{\omega_{k} r}{c_{2}}\right)-4 \frac{\omega_{k}}{c_{2} r} H_{1}^{(1)}\left(\frac{\omega_{k} r}{c_{2}}\right)+\right. \\
\left.4 \frac{\mu}{\lambda+2 \mu} \frac{\omega_{k}}{c_{1} r} H_{1}^{(1)}\left(\frac{\omega_{k} r}{c_{1}}\right)+\frac{12}{r^{2}}\left(H_{2}^{(1)}\left(\frac{\omega_{k} r}{c_{2}}\right)-\frac{\mu}{\lambda+2 \mu} H_{2}^{(1)}\left(\frac{\omega_{k} r}{c_{1}}\right)\right)\right]
\end{gathered}
$$




$$
\begin{aligned}
F_{22}\left(\mathbf{x}, \mathbf{y}, \omega_{k}\right)= & \frac{i \mu}{2}\left[\frac{\lambda^{2}}{(\lambda+2 \mu)^{2}}\left(\frac{\omega_{k}}{c_{2}}\right)^{2} H_{0}^{(1)}\left(\frac{\omega_{k} r}{c_{1}}\right)+2 \frac{\omega_{k}}{c_{2} r} H_{1}^{(1)}\left(\frac{\omega_{k} r}{c_{2}}\right)+\right. \\
& \left.2 \frac{\omega_{k}}{c_{1} r} \frac{\lambda}{\lambda+2 \mu} H_{1}^{(1)}\left(\frac{\omega_{k} r}{c_{1}}\right)-\frac{6}{r^{2}}\left(H_{2}^{(1)}\left(\frac{\omega_{k} r}{c_{2}}\right)-\frac{\mu}{\lambda+2 \mu} H_{2}^{(1)}\left(\frac{\omega_{k} r}{c_{1}}\right)\right)\right],
\end{aligned}
$$

where $H_{\beta}^{(1)}$ are the Hankel functions of first kind, $r=\left|x_{1}-y_{1}\right|$ is the distance between the points $\mathbf{x}$ and $\mathbf{y}$, and $c_{2}=\sqrt{\mu / \rho}$ is the velocity of the transverse wave in the material. The detailed expressions for the real and imaginary parts of integral kernels are given in [14].

\section{Crack closure, friction and iterative algorithms}

To include the contact interaction of the opposite crack's faces into account, the Signorini unilateral constraints must be imposed for the normal components of the contact force and the displacement vectors [18]

$$
\left[u_{n}(\mathbf{x}, t)\right] \geq 0, \quad q_{n}(\mathbf{x}, t) \geq 0, \quad\left[u_{n}(\mathbf{x}, t)\right] q_{n}(\mathbf{x}, t)=0, \quad \mathbf{x} \in \Omega, t \in[0 ; T]
$$

where $[\mathbf{u}(\mathbf{x}, t)]=\mathbf{u}^{(1)}(\mathbf{x}, t)-\mathbf{u}^{(2)}(\mathbf{x}, t)$ is the displacement discontinuity vector; and $\mathbf{q}(\mathbf{x}, t)$ is the contact force that arises in the contact region, which is unknown beforehand, changes in time under deformation of the material and must be determined as a part of solution. The contact region also depends on the frequency, magnitude and direction of the external loading complicating the problem even more and making it highly non-linear.

The contact constraints (22) ensure that there is no interpenetration of the opposite crack faces; the normal component of the contact force is unilateral and it is absent for any non-zero opening of the crack. Note that, due to the contact interaction the traction vector at the crack faces is the superposition of the initial traction caused by the incident load and the contact force. 
Furthermore, in order to take the friction of the opposite crack faces into account, we assume that the tangential components of the displacement discontinuities vector and vector of contact forces satisfy the Coulomb friction law:

$$
\begin{gathered}
\left|q_{\tau}(\mathbf{x}, t)\right|<k_{\tau} q_{n}(x, t) \Rightarrow \frac{\partial\left[\mathrm{u}_{\tau}(\mathbf{x}, t)\right]}{\partial t}=0 \\
\left|q_{\tau}(\mathbf{x}, t)\right|=k_{\tau} q_{n}(x, t) \Rightarrow \frac{\partial\left[\mathrm{u}_{\tau}(\mathbf{x}, t)\right]}{\partial t}=-\frac{q_{\tau}(\mathbf{x}, t)}{\left|q_{\tau}(\mathbf{x}, t)\right|}\left|\frac{\partial\left[\mathrm{u}_{\tau}(\mathbf{x}, t)\right]}{\partial t}\right|, \quad \mathbf{x} \in \Omega, t \in[0 ; T] .
\end{gathered}
$$

Thus, the opposite crack faces remain immovable with respect to each other in tangential direction while they are held by the friction force. However, as soon as the magnitude of the tangential contact forces reaches a certain limit, depending on the crack friction coefficient and the normal contact forces, the crack faces begin to move and the slipping effect occurs.

To solve the nonlinear contact problem for homogeneous isotropic cracked materials, four iterative algorithms were developed [18]. The comparative study of their convergence rates was carried out in [19].

The most effective and reliable algorithm used in [13, 14], and then applied for the interface cracks in [15] consists of the following main parts. In the first part of the algorithm, the solution of the elastodynamic problem for the cracked material neglecting the effect of the crack closure is obtained. Then the correction of the solution is performed applying the unilateral constraints with friction of the crack faces. During the iterative process, the Fourier coefficients are changing till the distribution of the vectors of displacements and contact forces satisfying the constraints is found.

In the current study the algorithm described above was successfully adapted for the considered case of transient loading and the convergence of the algorithm for different friction coefficients and different iterative coefficients has been analysed. 


\section{Numerical studies, analysis of the results and conclusions}

As a model numerical example we considered an incident pulse of the unit amplitude propagating in the normal direction to the interface of the linear crack with the length of $2 a$. The properties of the material (steel) were taken as follows: the Young's modulus $200 \mathrm{GPa}$, Poisson's ratio 0.25 , and the density $7800 \mathrm{~kg} / \mathrm{m}^{3}$.

As suggested in [17], the Heaviside impact pulse was approximated by the trapezoidal stress pulse

$$
\sigma(t)=\sigma^{*}\left\{\begin{array}{l}
\frac{t}{t^{*}}\left(H(t)-H\left(t-t^{*}\right)\right)+\left(H\left(t-t^{*}\right)-H\left(t-t^{*}-t_{d}\right)\right) \\
+\left(2-\frac{t-t_{d}}{t^{*}}\right)\left(H\left(t-t^{*}-t_{d}\right)-H\left(t-2 t^{*}-t_{d}\right)\right)
\end{array}\right\},
$$

where $c_{2} t^{*}=0.1$ and $c_{2} t_{d}=12$, and the latter one was approximated by the Fourier series for different numbers of the Fourier coefficients.

The dynamic stress intensity factor (opening mode only, as the shear mode is absent for the normal Heaviside pulse loading in the homogeneous material) normalized by the corresponding static value (obtained for the static tension of the unit magnitude) is given in Figure 1, where the model numerical solution by Gross and Zhang [20] is also presented.

As one can see the results are in a very good agreement. It shall be noted that the corrected solution is identical to the initial one as for the case of normal Heaviside tension pulse there is no contact interaction between the crack faces. The correction of the results for the case of normal cyclic loading was considered in details in our previous publications (e.g. [12-15]), thus in this study we will mainly focus on pure shear loading and effects of friction. 


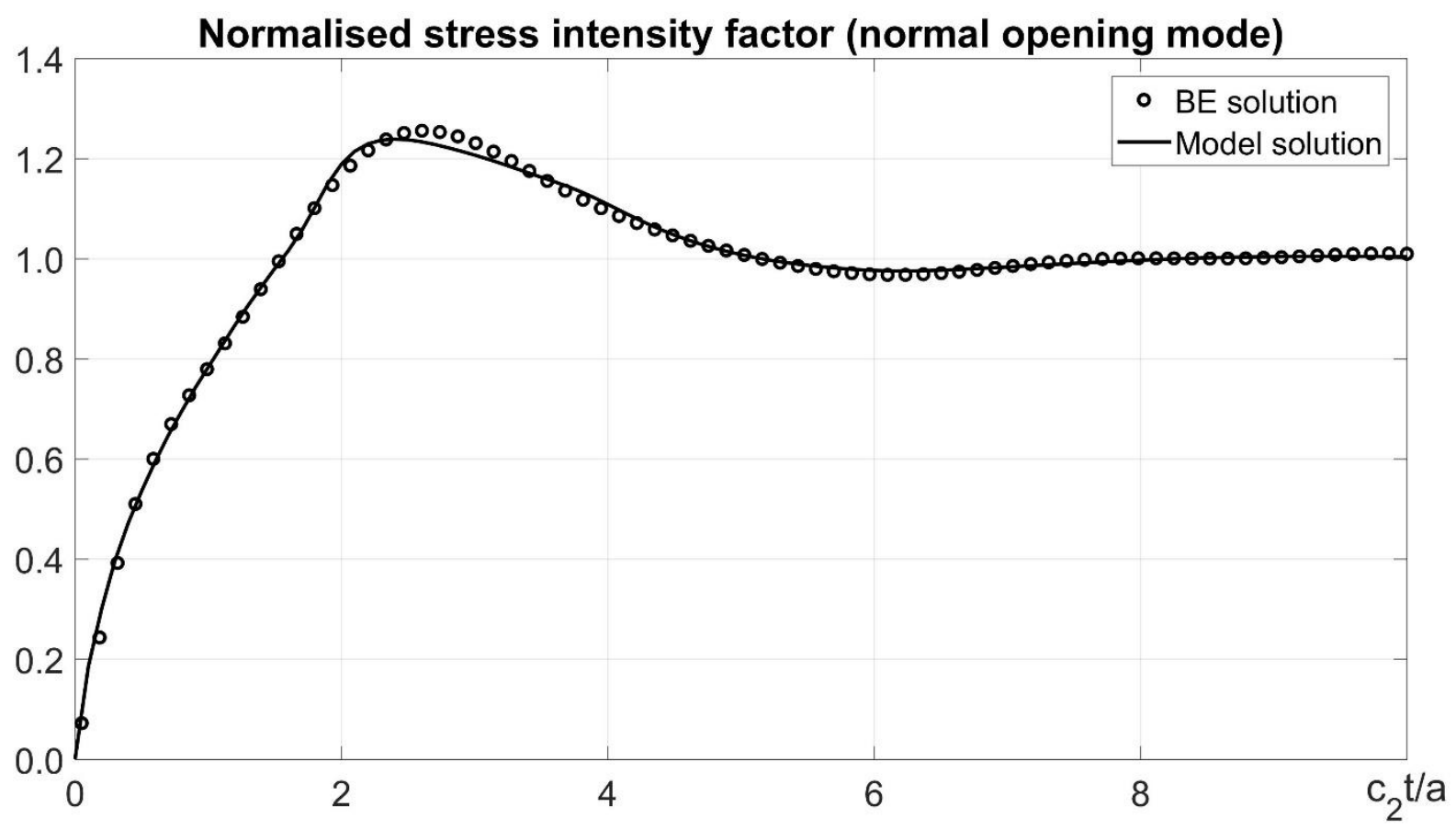

Figure 1. Stress intensity factor (normal opening mode) plotted against the normalized time, normal Heaviside pulse

The shear loading distributions presented in Figure 2 were considered, and the normal component of the contact force at the crack surface (to be used in the Coulomb friction law to hold the crack faces together) was assumed to be constant of unit magnitude.

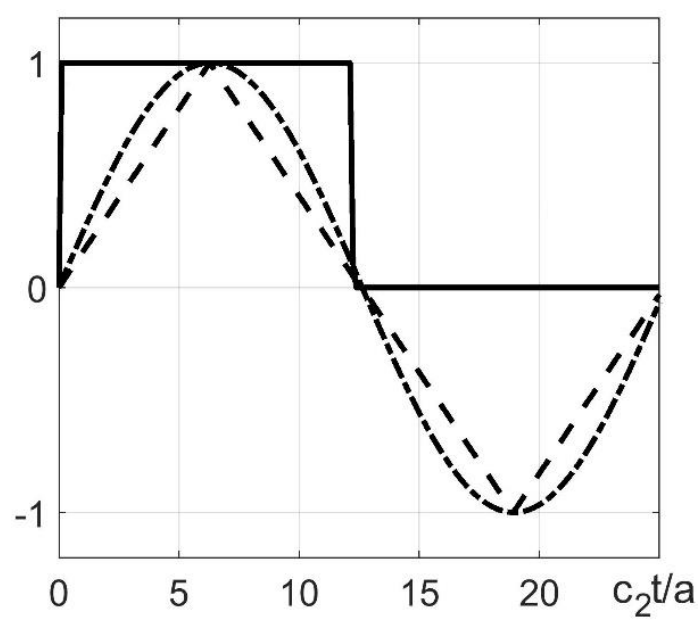

Figure 2. Loading shear pulses plotted against the normalized time 
The dynamic stress intensity factor (transverse shear mode) normalized by the corresponding static value (obtained for the static shear of the unit magnitude) are given in Figures 3-6, 9-14. The normalized distributions of the tangential components of the displacement jump and contact force on the crack surface for one of the pulses is given in Figures 7 and 8 .

Note that the results obtained for the harmonic loading (Figure 4) and the Heaviside shear pulse (Figure 14) are in a very good agreement with the model ones obtained accounting for the friction by the authors [14, 19], and by Sih, Embley and Ravera neglecting friction's effects [21], correspondingly.

Detailed analysis of the convergence rate of the iterative algorithm for different friction coefficients and different iterative coefficients $K_{T}$ is presented in Figures 3, 5, 9-13 (please see papers $[15,19]$ for the full description of the algorithm and the appropriate parameters introduced). One can clearly see the convergence of the iterative algorithm within the correction process with the convergence rate significantly decreasing with the rise of the friction coefficient (as expected, because more correction steps are needed). At the same time, it is possible to significantly increase the convergence rate using higher values of the iterative coefficient $K_{I}$ (see Figures 911 with $0.1<K_{T}<2.0$ ), but when the iterative coefficient exceeds a certain value, the process does not converge at all (Figure 12, results obtained for $K_{T}=20$ and $K_{T}=50$ ). Thus the results presented in the paper (Figures $3-8,13,14$ ) were obtained for the relatively low iterative coefficient $K_{T}=1$ with the proved convergence of the iterative algorithm.

It may be added that the detailed investigation of the solution's convergence with respect to the number of the boundary elements (and size of the elements in the vicinity of the crack's tips) and the number of time intervals has been presented in [14] 
and [15] for linear cracks in homogeneous and layered materials, thus in this paper we followed the recommendations suggested in [14]. Furthermore, a special attention must be paid to the number of Fourier coefficients used to approximate the harmonic solution (at least 10 coefficients are needed according to [15]), but on the other side, according to [17] for the impact (or sharp pulse) loading the number of Fourier coefficients shall be significantly higher (at least 30 coefficients to adequately approximate the Heaviside pulse). Thus, for the consistency in this paper we used 50 Fourier coefficients for all types of the loading.

It shall be also noted that the response time and the magnitude of the stress intensity factors clearly depend on the friction coefficient (Figure 14). As expected, the friction considerably decreases the maximal values of the corresponding dynamic stress intensity factors, and for the Heaviside shear pulse after the critical value of the friction coefficient (about 0.3 in the considered case) the maximum dynamic stress intensity factor coincides with the static stress intensity factor, which seems to be quite important from the practical point of view.

At the same time, the distribution of the normal contact forces (which was assumed here being constant to test the iterative algorithm) for any type of the loading different from the normal one would be quite complex $[13,14,20,22]$, causing the corresponding distribution of the tangential forces and displacements (very different from the ones presented in Figures 7 and 8), so the problem for oblique loading must be solved which seems to be the natural next stage of the research. Thus, the range of stress intensity factors (from "no friction" to "no sliding" scenarios) could be obtained for different directions of the loading. 


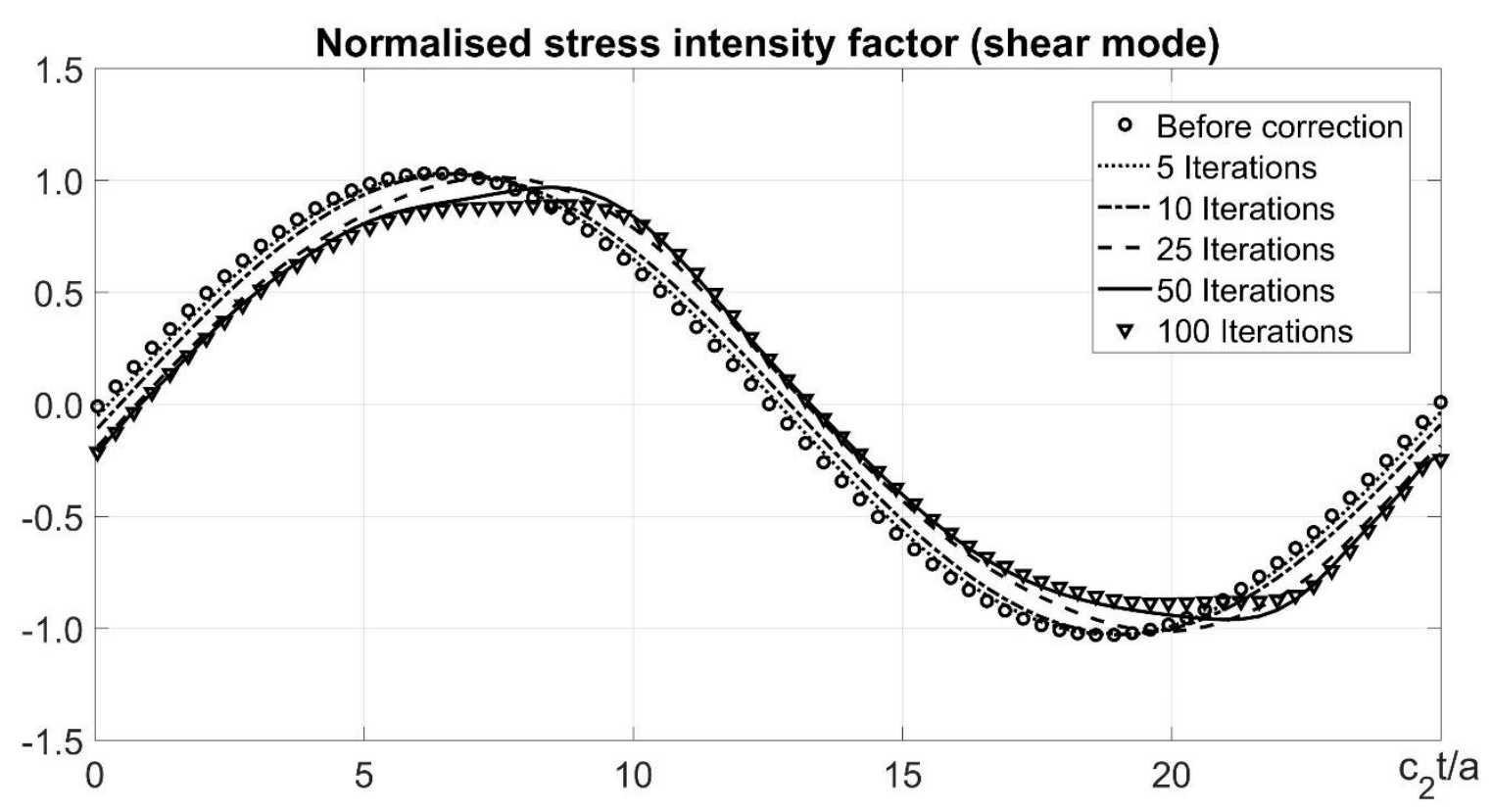

Figure 3. Stress intensity factor (shear mode) plotted against the normalized time for different numbers of iterations, harmonic shear, friction coefficient 0.2 .

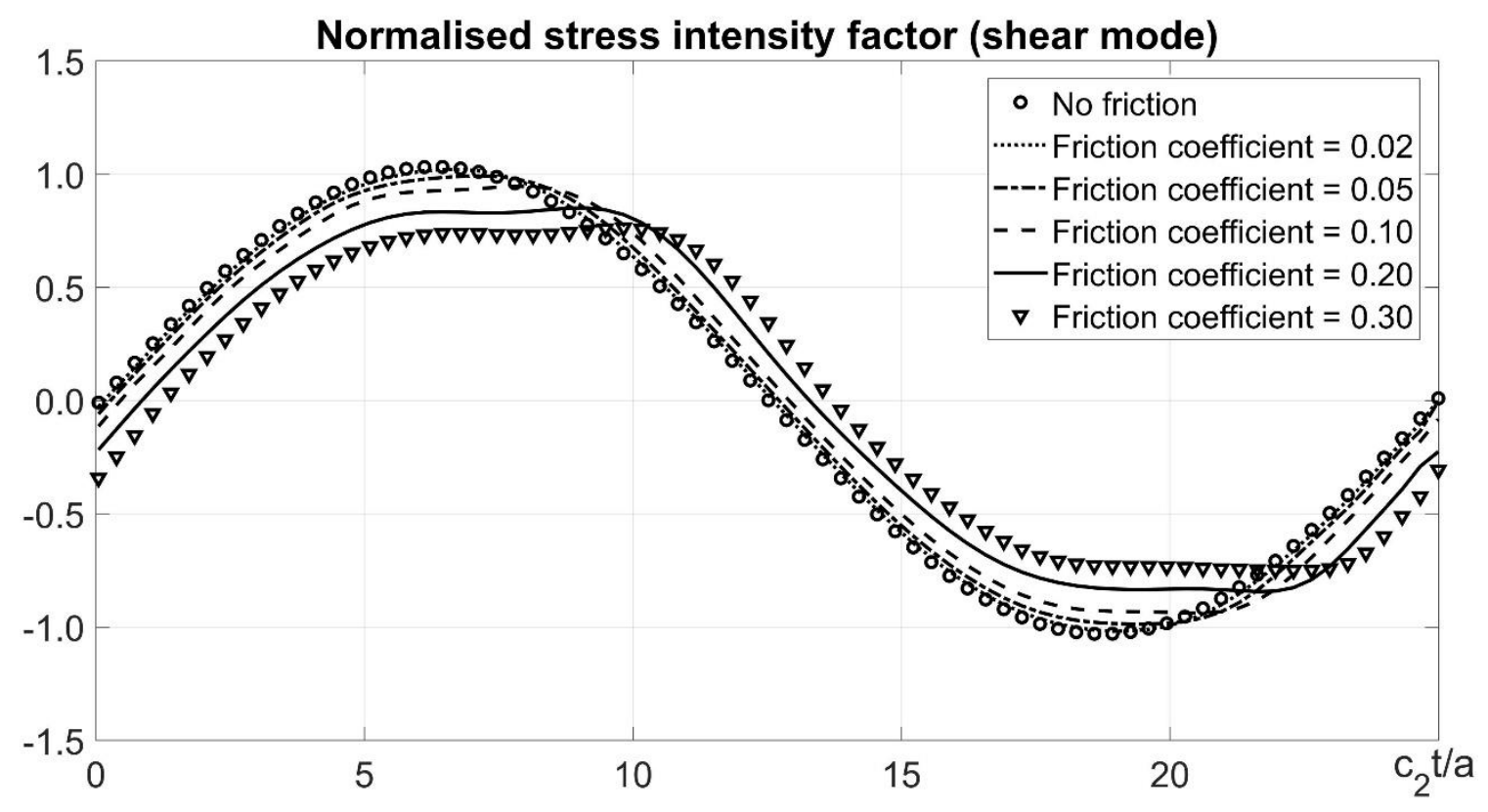

Figure 4. Stress intensity factor (shear mode) plotted against the normalized time for different friction coefficients, harmonic shear. 


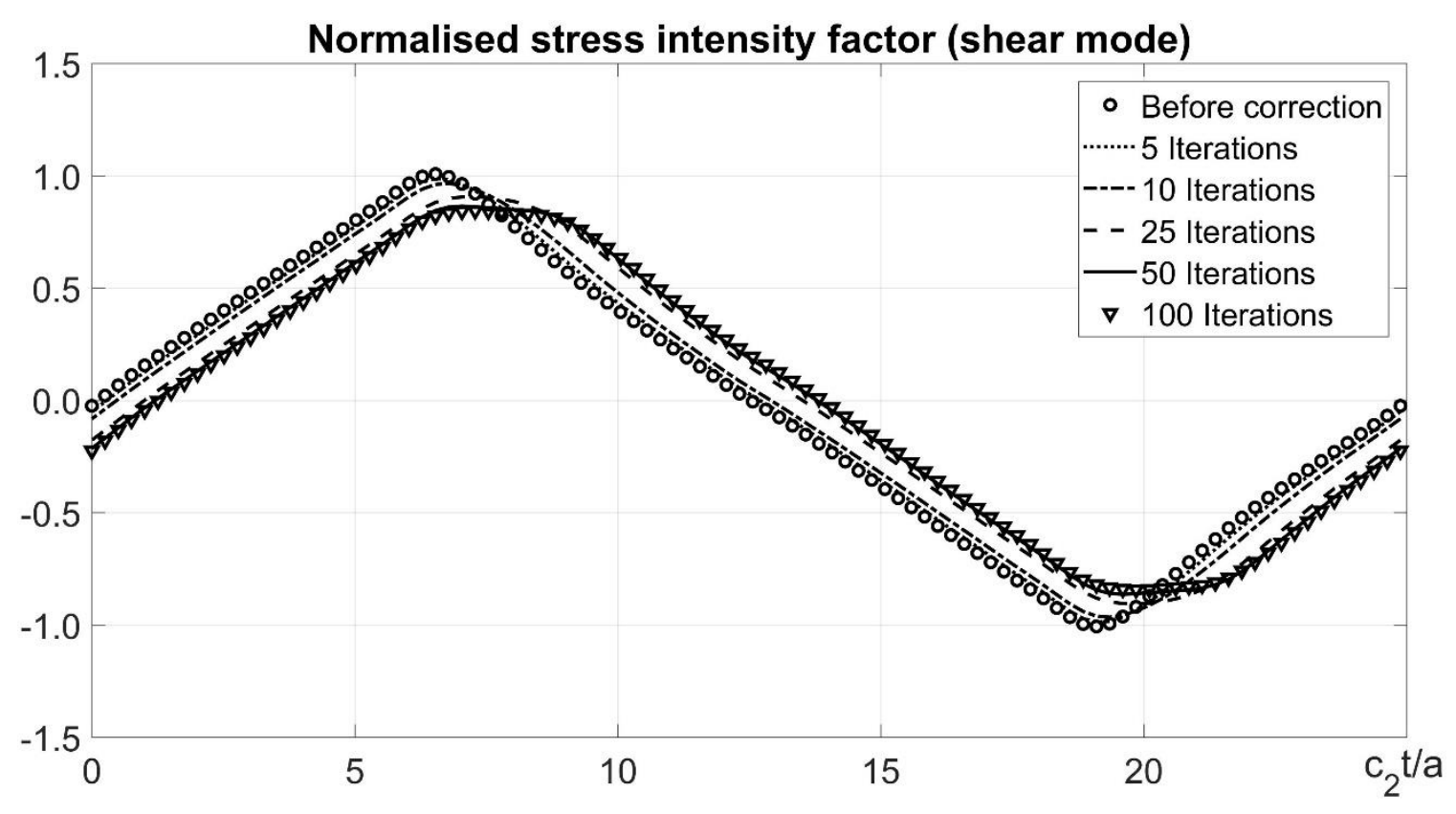

Figure 5. Stress intensity factor (shear mode) plotted against the normalized time for different numbers of iterations, "saw" shear pulse, friction coefficient 0.2 .

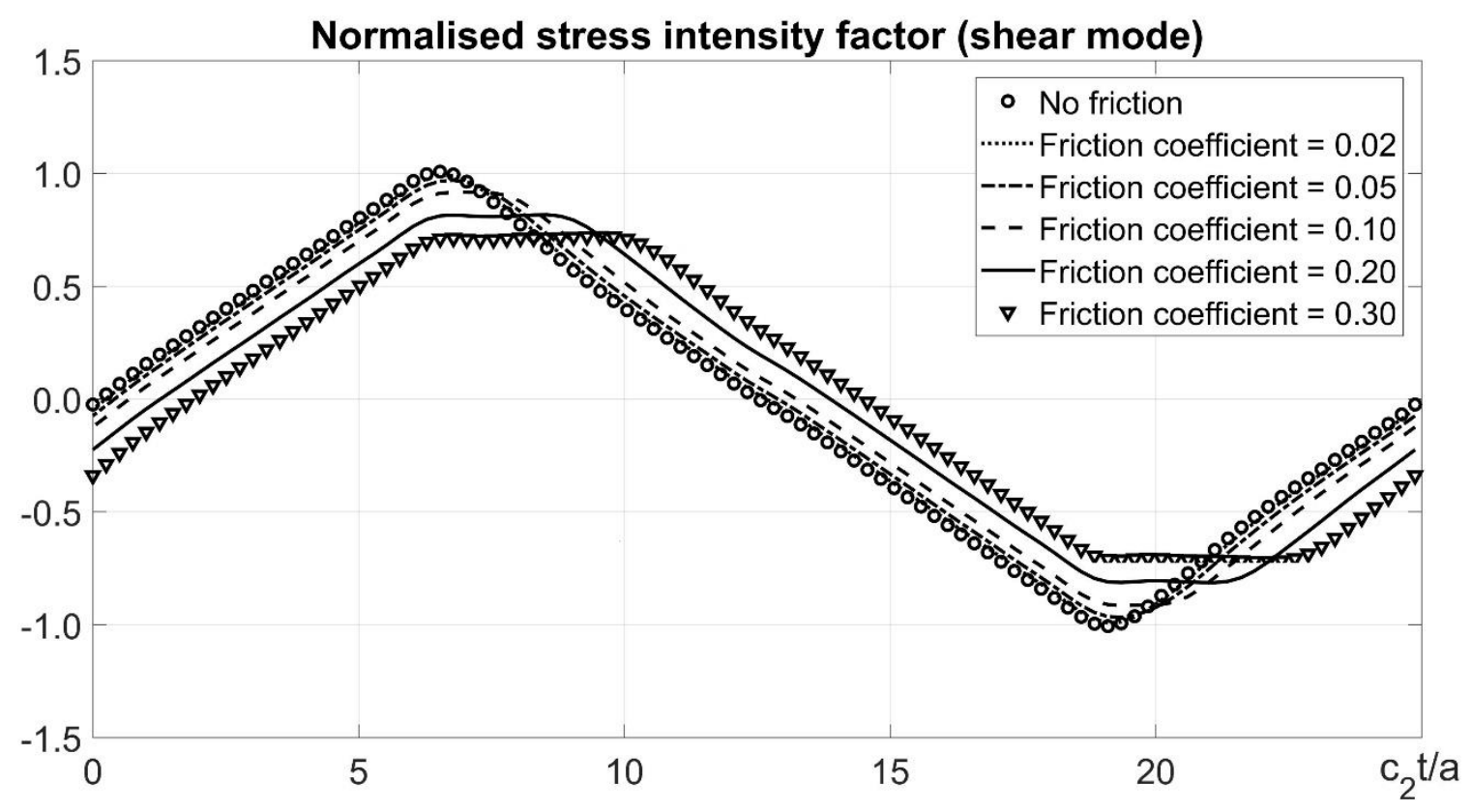

Figure 6. Stress intensity factor (shear mode) plotted against the normalized time for different friction coefficients, "saw" shear pulse. 


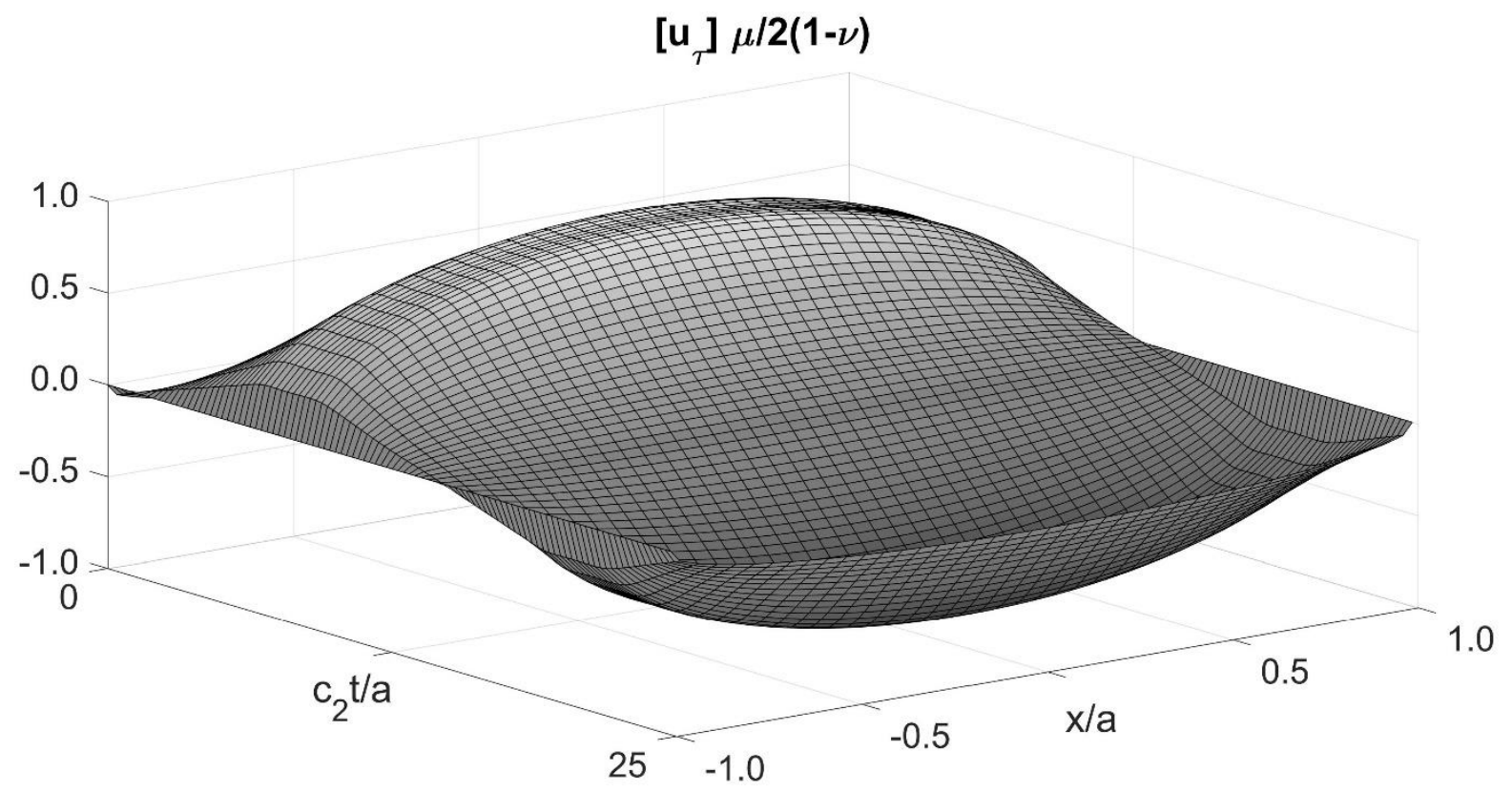

Figure 7. Normalised tangential displacements jump, "saw" shear pulse, friction coefficient 0.2 .

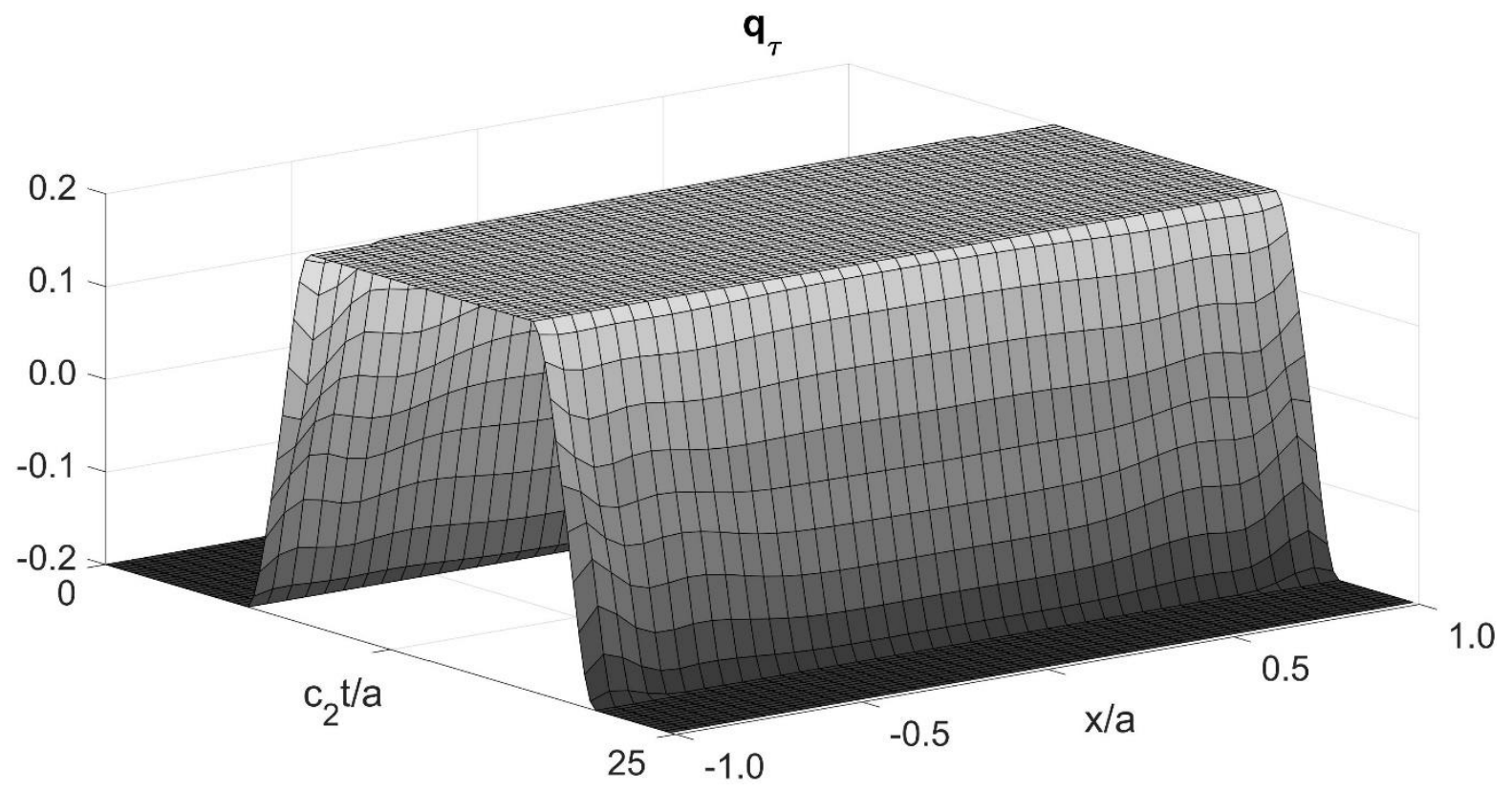

Figure 8. Tangential contact force, "saw" shear pulse, friction coefficient 0.2. 


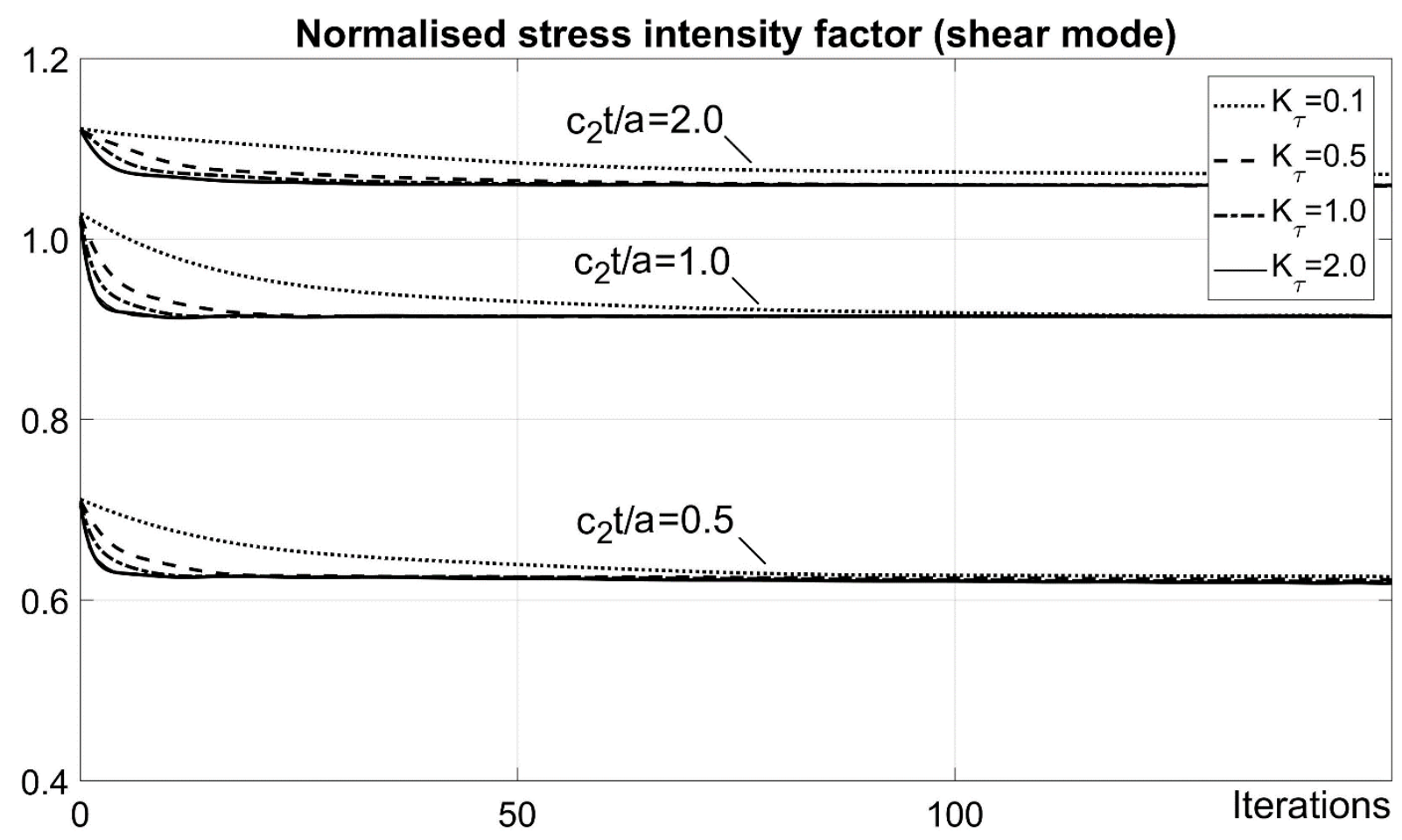

Figure 9. Stress intensity factor (shear mode) plotted against the number of iterations, Heaviside shear pulse, friction coefficient $=0.1$.

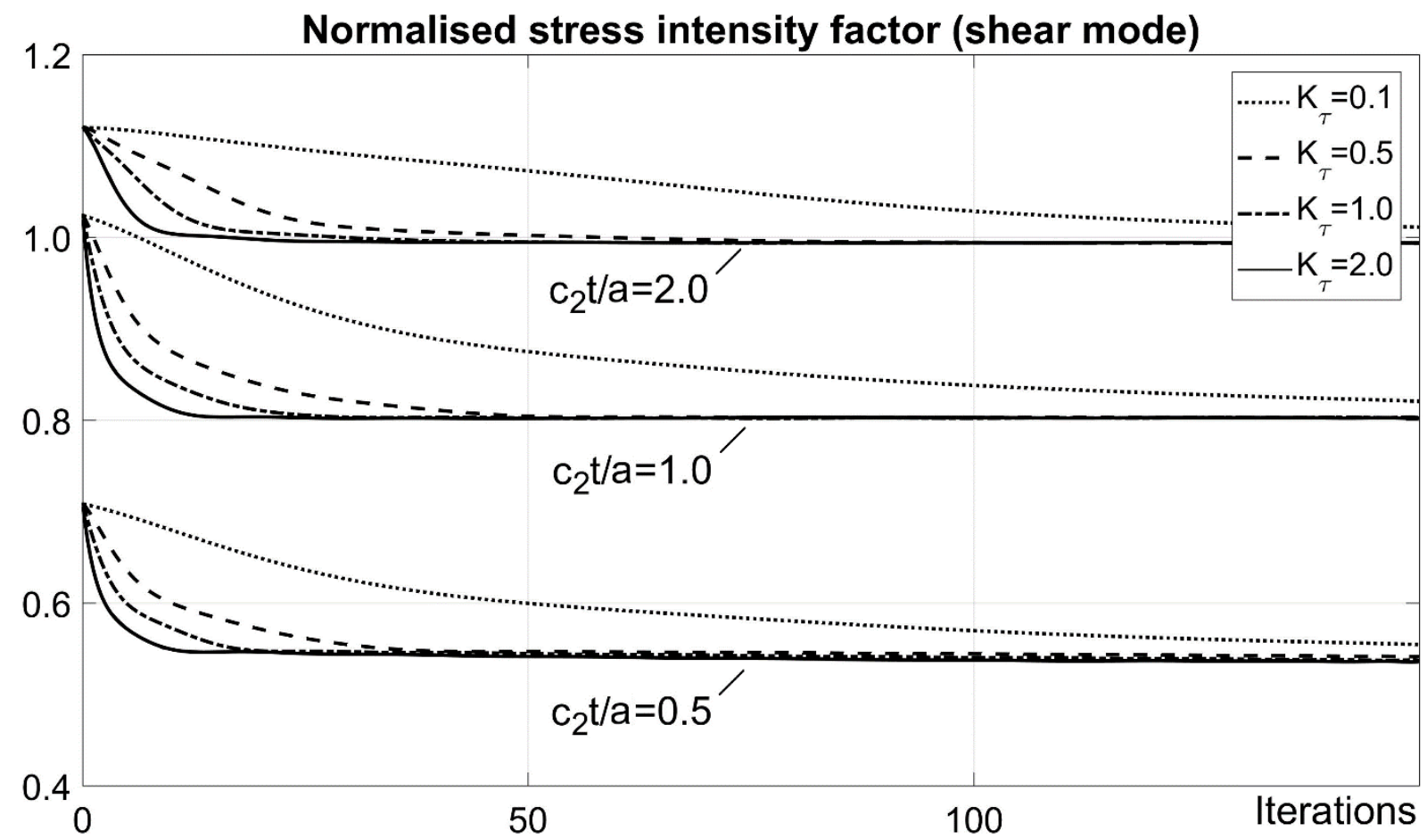

Figure 10. Stress intensity factor (shear mode) plotted against the number of iterations, Heaviside shear pulse, friction coefficient $=0.2$. 


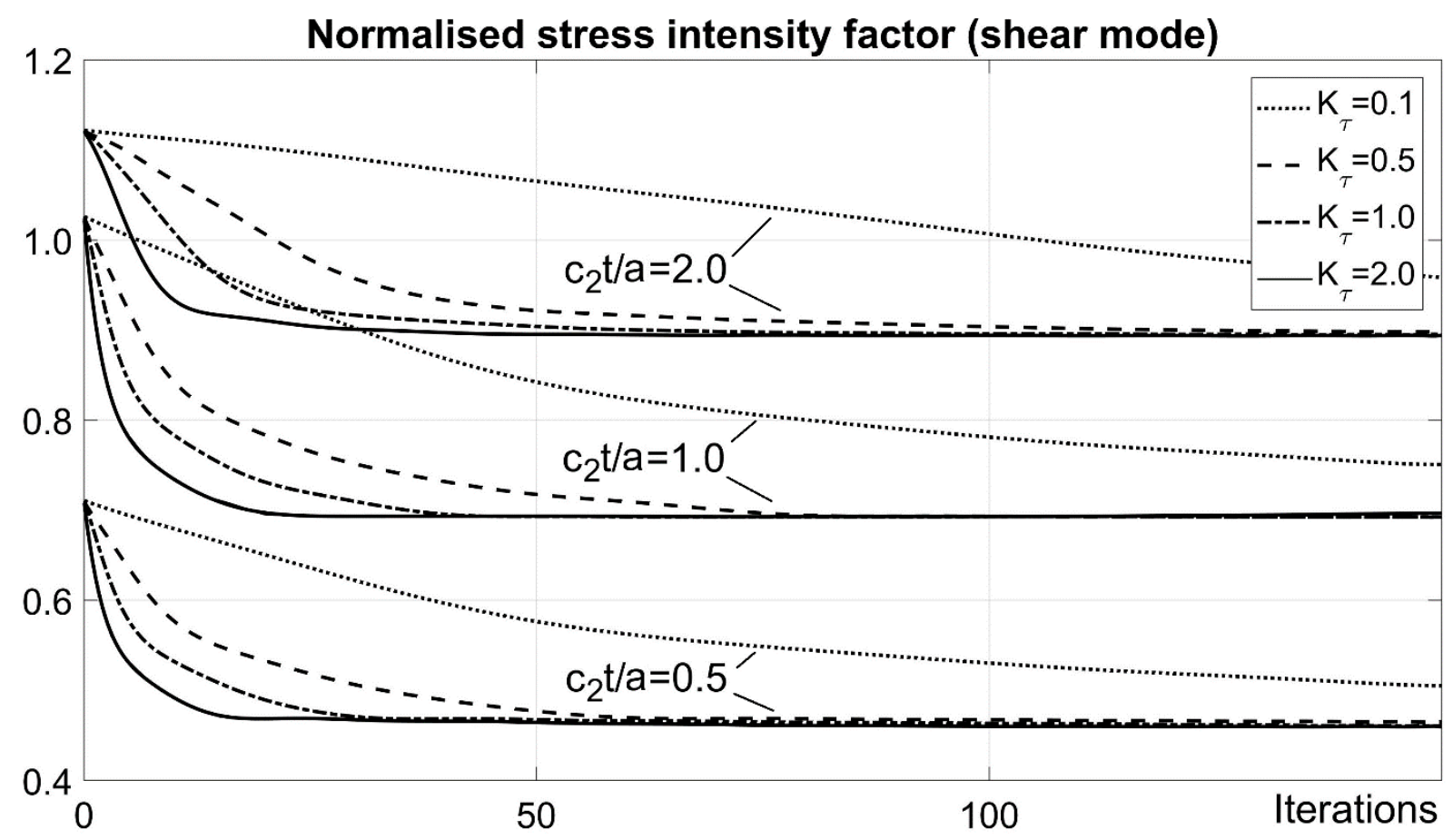

Figure 11. Stress intensity factor (shear mode) plotted against the number of iterations, Heaviside shear pulse, friction coefficient $=0.3$.

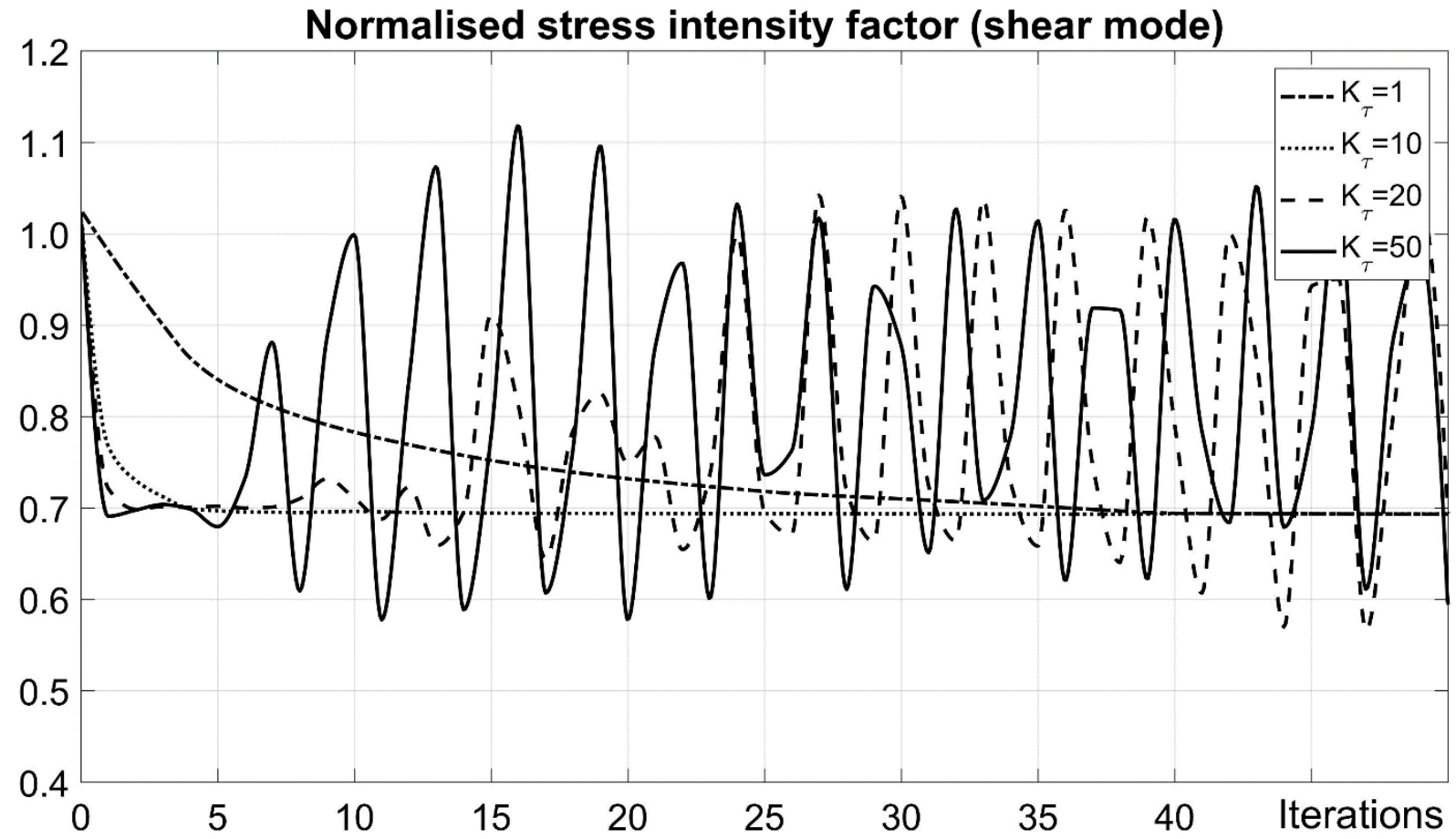

Figure 12. Stress intensity factor (shear mode) plotted against the number of iterations; Heaviside shear pulse; friction coefficient $=0.3$; normalized time $\mathrm{c}_{2} \mathrm{t} / \mathrm{a}=1.0$ 


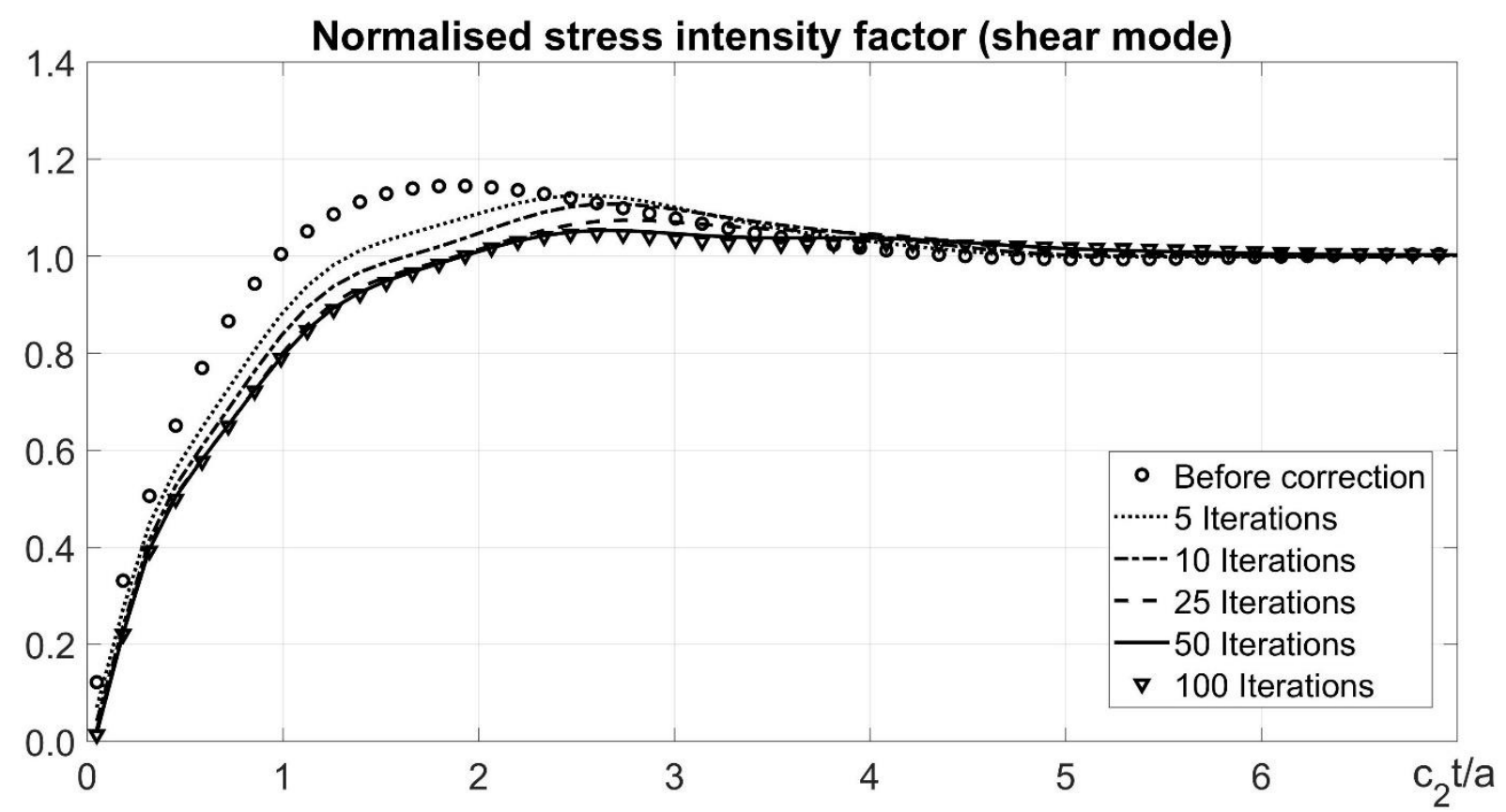

Figure 13. Stress intensity factor (shear mode) plotted against the normalized time, Heaviside shear pulse, friction coefficient 0.2.

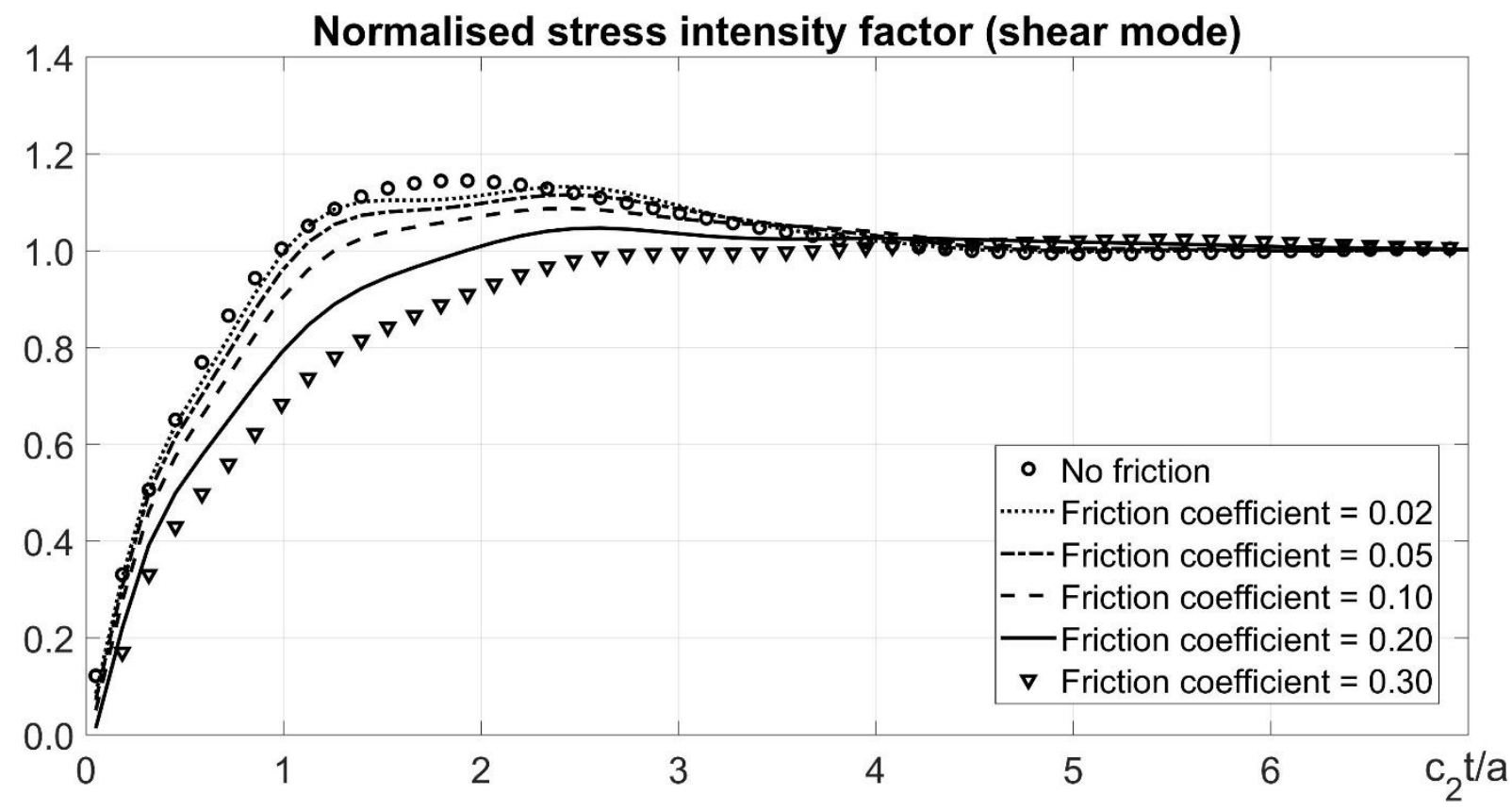

Figure 14. Stress intensity factor (shear mode) plotted against the normalized time for different friction coefficients, Heaviside shear pulse. 
Thus, the method developed and presented in the current paper may be combined with the boundary integrals presented in $[15,20,23-25]$ for interface cracks under different types of the loading. Furthermore, the approach can be extended to three-dimensional fracture mechanics problems similar to the ones considered in [2628] for homogeneous and layered cracked materials, with different approximation methods (for example, Galerkin methods used in [29]). In the future the method may be hopefully adapted to solve the problems in for the layered composites with cracked interlayers [30] and interface delaminations [31]. In all mentioned cases the iterative correction of the displacements and stresses to take the cracks closure and friction into account shall be an integral part of the solution procedure significantly improving the reliability of the solution obtained.

\section{References}

[1] Freund LB. The stress intensity factor due to normal impact loading of the faces of a crack. International Journal of Engineering Science. 1974; 12: 179-189.

[2] Ma CC, Hou YC. Theoretical analysis of the transient response for a stationary inplane crack subjected to dynamic impact loading. International Journal of Engineering Science. 1990; 28(12): 1321-1329.

[3] Ma CC, Hou YC, Transient analysis for antiplane crack subjected to dynamic loadings, Journal of Applied Mechanics. 1991; 58(3), 703-709.

[4] Wu KC, Chen JC. Transient analysis of collinear cracks under anti-plane dynamic loading. Procedia Engineering. 2011; 10: 924-929.

[5] Wu KC, Hou YL, Huang SM. Transient analysis of multiple parallel cracks under anti-plane dynamic loading. Mechanics of Materials. 2015; 81: 56-61. 
[6] Ayatollahi M, Monfared MM. Anti-plane transient analysis of planes with multiple cracks, Mechanics of Materials. 2012; 50: 36-46.

[7] Agrawal AK, Kishore NN. A study of free surface effects on through cracks under impact loading. Engineering Analysis with Boundary Elements. 2001; 25: 391-405.

[8] Garcia-Sanchez F, Zhang Ch, Sladek J, Sladek V. 2D transient dynamic crack analysis in piezoelectric solids by BEM. Computational Materials Science. 2007; 39: 179-186.

[9] Zhao P, Qin T, Zhang L. A regularized time-domain BIEM for transient elastodynamic crack analysis in piezoelectric solids. Engineering Analysis with Boundary Elements. 2015; 56: 145-153.

[10] Wuensche M, Zhang Ch, Garcia-Sanchez F, Saez A, Sladek J, Sladek V. On two hypersingular time-domain BEM for dynamic crack analysis in 2D anisotropic elastic solids. Computational Methods in Applied Mechanics and Engineering. 2009; 198: 2812-2824.

[11] Czekanski A, Zozulya V. Comparative study of time and frequency domain approaches in contact problem for the I-mode crack under harmonic loading. Engineering Analysis with Boundary Elements. 2018; 95: 200-214.

[12] Menshykov O, Guz IA. Effect of contact interaction of the crack faces for a crack under harmonic loading. International Applied Mechanics. 2007; 43(7): 809-815.

[13] Menshykov O, Guz I. Contact interaction of crack faces under oblique incidence of a harmonic wave. International Journal of Fracture, 2006; 139(1): 145-152.

[14] Menshykov OV, Menshykova MV, Guz IA. Effect of friction of the crack faces for a linear crack under an oblique harmonic loading. International Journal of Engineering Science. 2008; 46(5): 438-458. 
[15] Menshykova MV, Menshykov OV, Guz IA. An iterative BEM for the dynamic analysis of interface crack contact problems. Engineering Analysis with Boundary Elements. 2011; 35(5): 735-749.

[16] Menshykov V, Menshykov O, Guz IA. Interfacial crack between elastic half-spaces under harmonic loading. International Applied Mechanics. 2007; 43(8): 865-873.

[17] Menshykova MV, Menshykov OV, Guz IA, Wuensche M, Zhang Ch. A boundary integral equations method in the frequency domain for cracks under transient loading. Acta Mechanica. 2016; 227(11): 3305-3314.

[18] Guz AN, Zozulya VV. Elastodynamic unilateral contact problems with friction for bodies with cracks. International Applied Mechanics. 2002; 38(8): 895-932.

[19] Zozulya VV, Men'shikova MV. Study of iterative algorithms for solution of dynamic contact problems for elastic cracked bodies. International Applied Mechanics. 2002; 38(5): 573-577.

[20] Zhang Ch, Gross D. On wave propagation in elastic solids with cracks (Advances in Fracture Mechanics), WIT Press, 1997.

[21] Sih GC, Embley GT, Ravera RS. Impact response of a finite crack in plane extension. International Journal of Solids and Structures. 1972; 8(7): 977-993.

[22] Ostrik VI. Contact of faces of a rectilinear crack under complex loading and various contact conditions. Acta Mechanica. 2019; 230: 3741-3758.

[23] Menshykov OV, Guz IA, Menshykov VA. Boundary integral equations in elastodynamics of interface cracks. Philosophical Transactions of the Royal Society A: Mathematical, Physical and Engineering Sciences. 2008; 366(1871): 1835-1839. 
[24] Menshykov OV, Menshykov VA, Guz IA. Elastodynamics of a crack on the bimaterial interface. Engineering Analysis with Boundary Elements. 2009; 33(3): 294-301.

[25] Menshykova MV, Menshykov OV, Guz IA. Linear interface crack under plane shear wave. CMES - Computer Modelling in Engineering and Sciences. 2009; 48(2): 107-120.

[26] Mykhaskiv V, Stankevych V. Elastodynamic problem for a layered composite with penny-shaped crack under harmonic torsion, Zeitschrift für Angewandte Mathematik und Mechanik (ZAMM), 2019, DOI: 10.1002/zamm.201800193.

[27] Guz AN, Menshykov OV, Zozulya VV. Surface contact of elliptical crack under normally incident tension-compression wave. Theoretical and Applied Fracture Mechanics. 2003; 40(3): 285-291.

[28] Guz AN, Guz IA, Men'shikov AV, Men'shikov VA. Penny-shaped crack at the interface between elastic half-spaces under the action of a shear wave. International Applied Mechanics. 2009; 45(5): 534-539.

[29] Menshykov OV, Menshykova MV, Wendland WL. On use of the Galerkin method to solve the fracture mechanics problem for a linear crack under normal loading. International Applied Mechanics. 2005; 41(11): 1324-1329.

[30] Mykhaskiv V, Stankevych V, Zhbadynskyi I, Zhang Ch. 3-D dynamic interaction between a penny-shaped crack and a thin interlayer joining two elastic half-spaces. International Journal of Fracture. 2009; 159: 137-149.

[31] Golub M, Doroshenko O. Boundary integral equation method for simulation scattering of elastic waves obliquely incident to a doubly periodic array of interface delaminations. Journal of Computational Physics. 2019; 376: 675-693. 